\title{
Cultura gerencial: visión y alcances en el deporte universitario
}

Management culture: vision and scope in university sport

\author{
Summar Alfredo Gómez Barrios. ${ }^{1}$
}

Recibido: 10-02-2019 / Revisado: 15-02-209 /Aceptado: 04-03-2019/ Publicado: 14-06-2019

\begin{abstract}
.
https://doi.org/10.33262/cienciadigital.v3i2.5.531

The responsible involvement of a manager as a unifying factor in different organizational spaces with its people, implies investing in values, knowledge, traditions and other components of a managerial culture, which through conviction, persuasion and influence can ensure the reconfiguration of conceptions and meanings in the management and in the construction of common interests. Under this position, the purpose of this article is to analyze the vision and scope of the managerial culture of university sports in Ecuador. The research used as a methodological platform the qualitative approach, supported on a descriptive level and a field design. For the capture, processing and analysis of the data that emerged from interviews conducted, the study was based on grounded theory. The results confirm the interest in a managerial culture based on the knowledge and the desirable competences to direct the university sport, in order to guide the behavior of the people under his leadership and articulated with the university philosophy. The conclusions indicate the need for a university sport based on a management culture articulated with a value system and a managerial leadership that guarantees benefits to the university community.
\end{abstract}

Keywords: Management, managerial culture, vision, university sport.

${ }^{1}$ Universidad Iberoamericana del Ecuador, Ecuador, Centro de Investigación y Estudios del Deporte- CIED. sgomez@unibe.edu.ec-académico@deportes.ec 


\section{Resumen.}

El involucramiento responsable de un gerente como factor aglutinador en distintos espacios organizacionales con su gente, implica invertir en valores, conocimientos, tradiciones y demás componentes propios de una cultura gerencial, que mediante el convencimiento, la persuasión y la influencia puedan asegurar la reconfiguración de concepciones y significados en la gestión y en la construcción de intereses comunes. Bajo esta postura, el propósito de este artículo es analizar la visión y alcance de la cultura gerencial del deporte universitario en Ecuador. La investigación utilizó como plataforma metodológica el enfoque cualitativo, soportada en un nivel descriptivo y un diseño de campo. Para la captura, procesamiento y análisis de los datos que surgieron de entrevistas realizadas, el estudio se apoyó en la teoría fundamentada. Los resultados confirman el interés por una cultura gerencial basada en el conocimiento y las competencias deseables para dirigir el deporte universitario, a fin de orientar el comportamiento de las personas bajo su conducción y articulado con la filosofía universitaria. Las conclusiones indican la necesidad de un deporte universitario basado en una cultura gerencial articulada con un sistema de valores y en un liderazgo gerencial que garantice beneficios a la comunidad universitaria.

Palabras claves: Gerencia, cultura gerencial, visión, deporte universitario.

\section{Introducción}

Conducir una organización deportiva en los tiempos actuales es una tarea ardua y compleja. Supone gerenciar capacidades, recursos y relaciones con alto sentido de adaptación sin ceñirse a paradigmas tradicionales debido a que todos los procesos que intervienen en un sistema deportivo están en permanente cambio según las exigencias que plantean los avances científico-técnicos y a las constantes transformaciones sociales, especialmente en fenómenos deportivos, tal como lo expresa Gómez (2017a, p. 36) cuando sostiene que el deporte es “... uno de los fenómenos sociales y culturales más importantes del nuevo milenio, tanto en su vertiente de espectáculo de masas como de práctica libre y voluntaria del ciudadano...”. Este enfoque resulta apropiado para comprender el compromiso que se adquiere al gerenciar el deporte y los límites dentro de los cuales se mueven estas organizaciones, que aunque difieren -según su finalidad- en su objetivo principal, en las actividades que desarrollan, en el nivel donde operan y en las entidades físicas que representan, coinciden en responder al fin superior de promover y desarrollar el deporte.

Estos razonamientos también dejan su huella en la gerencia del deporte universitario, el cual debe conducir a la satisfacción de las necesidades deportivas de los miembros de la comunidad universitaria, estimando políticas y programas acordes a las necesidades reales, 
garantizando atención integral al deportista universitario y manteniendo relaciones con el entorno académico. Total, un modelo de gestión del deporte universitario que preste un servicio eficaz y eficiente a toda la comunidad universitaria para la promoción y desarrollo del deporte mediante procesos participativos, ya sean hacia la recreación, hacia la formación o hacia la competición.

Por tanto, saber administrar, dirigir, gestionar el deporte universitario en el contexto de la sociedad actual exige un gerente con el conocimiento necesario para el ejercicio del rol, el cual debe ser adquirido en la academia y producto también de la experiencia en el deporte, para que luego pueda ser desarrollado, distribuido, utilizado, preservado, medido. En resumidas cuentas, el conocimiento es "una capacidad humana, basada en la experiencia, que tiene como fin transformar la información en decisiones y acciones concretas" (Santillán, 2010, p. 23), razón por la cual debe ser debidamente gestionado para que sea transferido y utilizado por otras personas.

Adicionalmente, la práctica gerencial debe complementar este conocimiento con un conjunto de competencias, que en sí mismas son habilidades blandas para resolver con eficacia y autonomía diversas situaciones -que en ocasiones implican riesgos e incertidumbre- basadas en las mejores decisiones (Beneitone, 2007, citado por Domínguez, 2018). Significa entonces, una gerencia del deporte universitario fundamentada en un saber qué (conocimiento) y en un saber cómo (competencias) en un mundo globalizado, cambiante y altamente competitivo.

Así pues, si en un proceso gerencial se manejan aquellas complejidades que comúnmente encierra la dinámica deportiva como se ha venido señalando, las acciones de un gerente del deporte universitario deberán encaminarse en lograr el apoyo de terceras personas. A tal efecto, Rusque (2008) sugiere que un gerente debe tener la capacidad para coordinar las acciones del recurso humano y maniobrar con éxito la complejidad. En consideración a ello, debe ser buen negociador y poseer potencial de liderazgo, cuyas características están determinadas por entender el contexto local y global, dar un lugar central al recurso humano, tomar rápidas decisiones y poseer gran capacidad de trabajo. Este planteamiento se complementa con el siguiente:

... no se puede lograr aquello que se ha propuesto (...) si las personas dentro de estas instituciones ejercen sus labores de forma individual; más bien deben funcionar bajo un sistema de relaciones alineadas con objetivos claros, con propósitos compartidos y con beneficios comunes (Gómez, 2018, p. 36).

Se deduce entonces, que buena parte de las acciones gerenciales deben orientarse hacia el empleo de mecanismos que propendan a motivar, inspirar, incentivar, convencer, persuadir e influenciar a las personas, en otros términos a liderar el talento humano bajo su dirección, 
configurando sólidas relaciones para sumar esfuerzos en conjunto articulados con la filosofía organizacional. Son mecanismos que habitualmente se admiten como competencias gerenciales. En esta misma dirección, Aguilar (2014) sostiene que el manejo y la administración del talento humano es una responsabilidad en un gerente que pudiera no ser tan fácil, dado que en la personalidad de ese talento subyacen emociones, sentimientos, valores, actitudes, hábitos y costumbres, siendo imperativo la influencia que debe ejercer el gerente para alinear esos rasgos de personalidad -convertidos en comportamientos y acciones- con los objetivos y metas organizacionales.

Sobre la base de estas consideraciones, se puede decir que el deporte universitario en Ecuador demanda un comportamiento gerencial que esté soportado en el conocimiento -susceptible de ser evaluado para incorporar nuevos aprendizajes-, en la experiencia acumulada y en aquellas competencias deseables; factores que sin duda permitirían el aseguramiento de un desempeño exitoso. Para la consecución de tales propósitos y su continuidad en el tiempo, el deporte universitario debe apalancarse en una firme cultura gerencial que combine aquellas creencias, valores, costumbres, actitudes, en suma una simbología gerencial orientada a materializar de forma efectiva un comportamiento tendiente a alcanzar resultados de excelencia. En este sentido, a continuación se citan algunos referentes que han reflexionado en torno a la cultura:

“... marco de referencia compartido y aceptado por el grupo de personas de una organización, orientando la manera de pensar y actuar (...), donde se incluyen aspectos intangibles (actitudes, valores, emociones) y tangibles vinculados a las operaciones cotidianas" (Gómez, 2018, pp. 36-37).

“... las creencias, valores, normas, marcos de referencia no son palpables, solo se logra evidenciar su influencia (...) a través de sus miembros, determinando de este modo, que la cultura influye en el comportamiento de los integrantes de la organización" (Montoya, 2014, citado por Rivera, Carrillo, Forgiony, Nuván, y Rozo, 2018, p. 28).

"Es la programación colectiva de la mente que distingue a los miembros de un grupo humano de otro. En este sentido, cultura incluye sistemas de valores, y estos valores forman parte de las células que constituyen la cultura" (Hofstede, 1984, citado por Taylor, 2007, p. 137).

“... cultura puede definirse como la suma combinada de las opiniones individuales, los valores compartidos y las normas de los miembros de la organización" (Swiringa y Wierdma, 1995, p. 13).

Resulta evidente de estas definiciones inherentes al término "cultura", la recurrente presencia del descriptor "valores". Visto así, los valores emergen como categoría central de la cultura, 
en el cual giran los demás descriptores como las creencias, las normas, las actitudes, las emociones. En cualquier caso, son elementos atribuidos a la cultura los cuales son aceptados y compartidos por las personas que hacen vida en una organización orientando su comportamiento para garantizar resultados.

Por otra parte, autores como Echeverría (2009), Taylor (2007) y Robbins (2004), coinciden en que la cultura fomenta el compromiso de la gente, eleva la coherencia en el comportamiento, reduce la ambigüedad, moldea multitud de factores de comunicación, indica cómo hacer las cosas, define qué es lo importante. En fin, una cultura es una manera de hacer las cosas en el aquí y ahora con dirección hacia el futuro.

Dados los argumentos que anteceden, la investigación se focalizó en analizar la visión y alcance de la cultura gerencial del deporte universitario en Ecuador, vista la cultura gerencial como aquella centrada en indicar “... cuál es el modo esperado de pensar y actuar frente a situaciones concretas (...) no atiende soluciones puntuales (...), pero señala las prioridades y preferencias globales que orientan a los actores de la organización" (Gómez, 2017b, p. 57), definición que con precisión engloba la intención del autor de este estudio, en aras de conocer desde la perspectiva de los actores entrevistados cuál es su percepción en torno al fenómeno objeto de tratamiento, a partir de tres categorías de análisis: (1) Perfil Gerencial, (2) Características de la Cultura Organizacional y (3) Concepción de las Cultura Gerencial.

\section{Metodología}

Esta investigación, cuyo objetivo fue analizar la visión y alcance de la cultura gerencial del deporte universitario en Ecuador, se fundamentó en el paradigma cualitativo con la intención de obtener datos desde la perspectiva de estos actores acerca de la percepción que tenían del objeto de estudio. En efecto, referentes teóricos como Martínez (2009) y Rusque (2007) sostienen que el paradigma cualitativo busca describir, analizar e interpretar las experiencias, actitudes, creencias y pensamientos de las personas en situaciones concretas, descubriendo el significado de aquello que es de naturaleza subjetiva, motivo por el cual “... el sujeto se reintegra (...) a la cultura y deja estar fuera, por debajo, por arriba, en otra parte" (Morin, 1989, p. 255). Bajo la premisa de estos autores, la investigación profundizó en aquella realidad que se encuentra adentro, en la consciencia del sujeto, donde se admite la subjetividad para descubrir y comprender cómo los actores sociales vislumbran el fenómeno a partir de sus valoraciones, actitudes y creencias, aspectos que fueron de cardinal importancia en este estudio.

En razón de lo anterior, la investigación adoptó un nivel descriptivo a fin de caracterizar aquellos conceptos que iban emergiendo y conocer su relación con las categorías seleccionadas, que mediante su ordenamiento en unidades hermenéuticas pudieron analizarse e interpretarse. Al mismo tiempo, se empleó un diseño de campo con el propósito de tener una proximidad con los actores sociales dentro del contexto estudiado (Arias, F., 2016), 
facilitando la sistematización operativa y metodológica del conjunto de datos que fueron surgiendo.

En este tenor, la sistematización del conjunto de ideas, conceptos y representaciones que se derivaron de la visión que tenían los actores consultados respecto al fenómeno objeto de tratamiento, se realizó bajo el método de la teoría fundamentada. Según Strauss y Corbin (2002), la teoría fundamentada busca generar aquel conocimiento que surge a partir de los datos que se presentan en los procesos sociales básicos y que son relevantes dentro de una particular área de estudio. Visto así, en esta investigación como proceso social están presentes aspectos como el rol que ocupan los actores sociales, el uso de un lenguaje particular, las simbologías y los significados que se derivan de sus relaciones -además del contexto donde ellos operan-, cada uno a su vez con necesidades específicas. De ahí que se entrevistaron a personas clave y con suficiente relevancia en cuanto a su conocimiento en materia deportiva, experiencia gerencial y/o docente en deporte universitario, y vivencia en la práctica sistemática y continua en alguna especialidad deportiva.

Así pues, el método de la teoría fundamentada se soportó de forma sistematizada en cuatro factores:

- El método comparativo constante: Implicó codificar los datos mediante la conceptualización, categorización y relaciones entre sí, los cuales fueron depurados mediante su comparación permanente.

- El muestreo teórico: Consistió en seleccionar nuevos sujetos para la obtención de nuevos datos y ampliar aquellos que fueron desarrollados.

- La codificación abierta: Proceso analítico que se basó en identificar conceptos mediante códigos vivos o sustantivos, que luego se clasificaron en categorías seleccionadas según su afinidad en términos de propiedades.

- La codificación axial: Proceso analítico que buscó cruzar los conceptos con las categorías para determinar el tipo su relación.

Con base en estos factores, este proceso de análisis sustentado en el método de la teoría fundamentada (ver figura 1) se inició con el muestreo teórico al seleccionar un entrevistado, y una vez transcrita la entrevista se hizo su conversión en unidad hermenéutica a objeto de iniciar el proceso de análisis en codificación abierta para identificar conceptos, etiquetarlos y clasificarlos en categorías y seguidamente aplicar el análisis en codificación axial para determinar el tipo de relación entre las etiquetas conceptuales y las categorías. 
Figura 1. Proceso de análisis sustentado en el método de la teoría fundamentada.

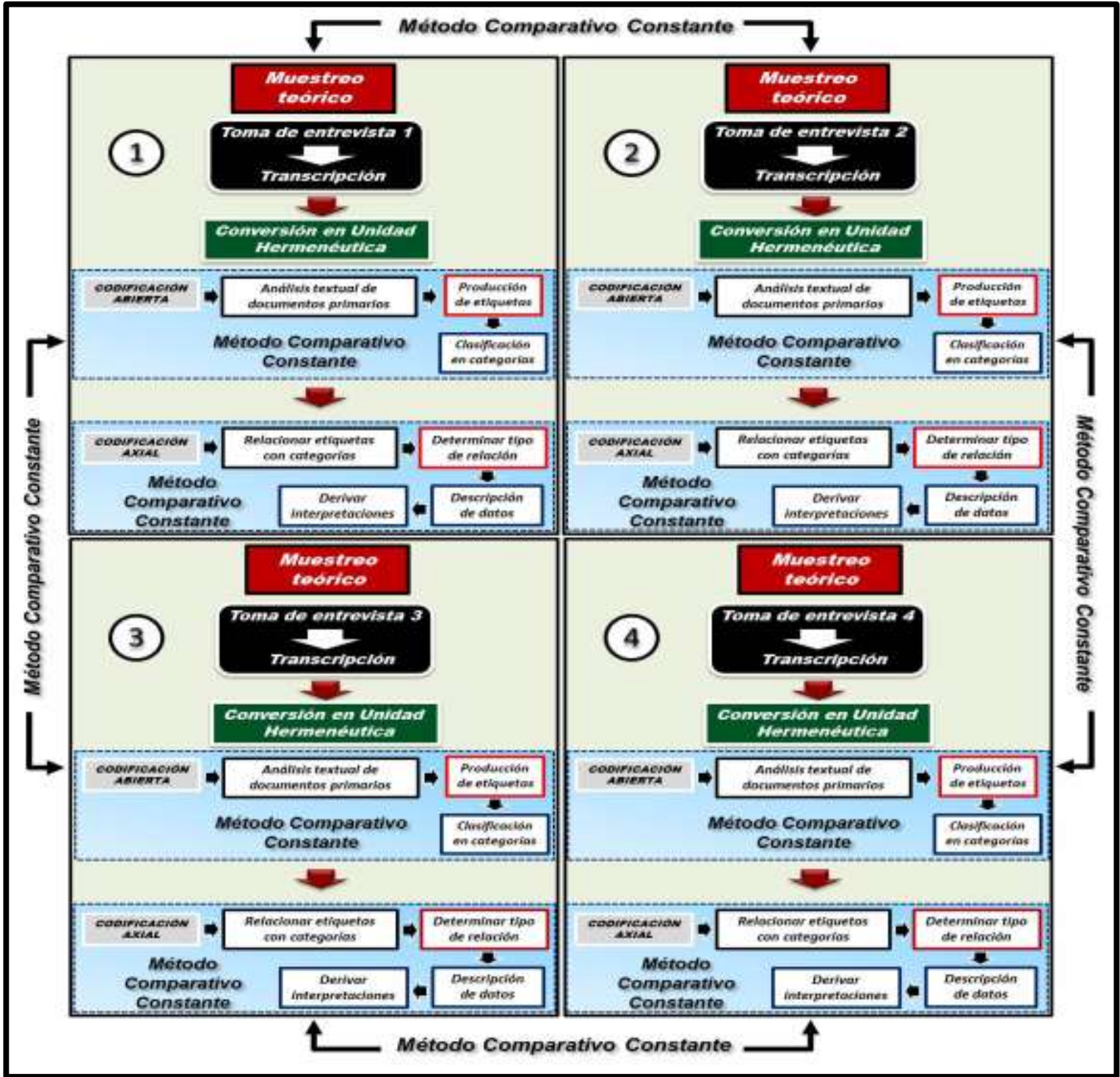

Fuente: Adaptado de Gómez (2018).

Apenas culminado este análisis, se realizó el siguiente muestreo teórico y se siguió el mismo procedimiento de forma sucesiva hasta llegar a la saturación teórica, mediante la cual no surgieron nuevos datos o nueva información para ser analizada, dando lugar a un total de cuatro entrevistados (ver tabla 1). Cabe señalar que desde el inicio del proceso de análisis, los datos que iban emergiendo eran comparados permanentemente dentro de la misma unidad hermenéutica y con otras unidades hermenéuticas, a los efectos de depurar la información obtenida y garantizar la congruencia necesaria en el ordenamiento de los datos en cuanto a conceptualización, categorización y relaciones entre sí. 
Tabla 1. Actores entrevistados con base en su conocimiento, experiencia y vivencia en el deporte.

\begin{tabular}{|c|c|c|}
\hline ENTREVISTADO & CARGO & RESUMEN SUCINTO \\
\hline $\begin{array}{l}\text { ANDRÉS } \\
\text { ZALDUMBIDE }\end{array}$ & $\begin{array}{l}\text { Profesor del Instituto } \\
\text { Tecnológico de Pichincha }\end{array}$ & $\begin{array}{l}\text { Licenciado en Ciencias de la Actividad Fisica, Deportes y Recreación. } \\
\text { Maestria en Gerencia Deportiva de FUNIBER - Fundación Universitaria } \\
\text { Iberoamericana de México y Maestria en Docencia Universitaria en la } \\
\text { ESPE. Ex entrenador y ex atleta. }\end{array}$ \\
\hline $\begin{array}{l}\text { PAOLA } \\
\text { HERRERA }\end{array}$ & $\begin{array}{l}\text { Coordinadora de la Carrera de } \\
\text { Entrenamiento Deportivo del } \\
\text { Instituto Tecnológico Superior } \\
\text { Libertad } \\
\end{array}$ & $\begin{array}{l}\text { Licenciada en Ciencias de la Actividad Física, Deporte y Recreación. } \\
\text { Maestria en Entrenamiento Deportivo de la Universidad de las } \\
\text { Fuerzas Armadas. } 30 \text { años vinculada al baloncesto como atleta y } \\
\text { dirigente. }\end{array}$ \\
\hline $\begin{array}{c}\text { JUAN } \\
\text { FRANCISCO } \\
\text { AGUINAGA }\end{array}$ & $\begin{array}{l}\text { Coordinador de actividades } \\
\text { extracurriculares de las } \\
\text { Universidad de Las Américas }\end{array}$ & $\begin{array}{l}\text { Ex jugador de futbol profesional con experiencia en equipos } \\
\text { nacionales y europeos, además de integrante de la selección de } \\
\text { Ecuador, Director técnico de futbol en distintas categorias y sectores } \\
\text { del deporte. }\end{array}$ \\
\hline $\begin{array}{l}\text { MIGUEL ÁNGEL } \\
\text { CARTAYA }\end{array}$ & $\begin{array}{l}\text { Coordinador de la Carrera de } \\
\text { Educación Fisica de la } \\
\text { Universidad Laica Eloy Alfaro } \\
\text { de Manabi }\end{array}$ & $\begin{array}{l}\text { Docente e investigador en la misma institución. Licenciado en } \\
\text { Educación Fisica y Pho en Ciencias de la Educación. Vinculado a la } \\
\text { Gimnasia en Venezuela por } 40 \text { años como atieta y luego como } \\
\text { dirigente deportivo. }\end{array}$ \\
\hline
\end{tabular}

Fuente: Elaboración propia.

\section{Resultados}

\section{Tendencias en codificación abierta y codificación axial}

A los fines de realizar una ajustada interpretación de los hallazgos encontrados con base en las evidencias que surgieron en cada una de las unidades hermenéuticas en torno a la visión y alcance de la cultura gerencial del deporte universitario en Ecuador, se procedió a consolidar la totalidad de los datos a fin de determinar las tendencias, en primera instancia en codificación abierta y posteriormente en codificación axial.

\section{Tendencias en codificación abierta}

Para estimar las tendencias en codificación abierta, primeramente se logró identificar conceptos de interés producto de la fragmentación de datos del análisis textual de las entrevistas; conceptos que se etiquetaron y clasificaron en tres categorías previamente seleccionadas en atención a sus propiedades o atributos, como son:

- Perfil Gerencial: Rasgos asociados al ejercicio gerencial que aseguran comportamientos deseables para un desempeño exitoso.

- Características de la Cultura Organizacional: Atributos de un sistema determinado por el conjunto de relaciones interpersonales existentes en la organización en conexión con la filosofía organizacional.

- Concepción de la Cultura Gerencial: Postura que se tiene respecto a la manera de ver y concebir el quehacer gerencial desde la perspectiva de una cultura orientada a generar cambios personales, grupales y organizacionales. 
Sobre la base de estas consideraciones, el análisis realizado en esta modalidad de codificación produjo un total de 75 etiquetas conceptuales, cuya distribución se inclinó hacia la categoría Perfil Gerencial y Concepción de la Cultura Gerencial con 36 y 32 etiquetas respectivamente. La categoría Características de la Cultura Organizacional se mantuvo distante con 7 etiquetas conceptuales (ver figura 2).

Figura 2. Tendencias en codificación abierta.

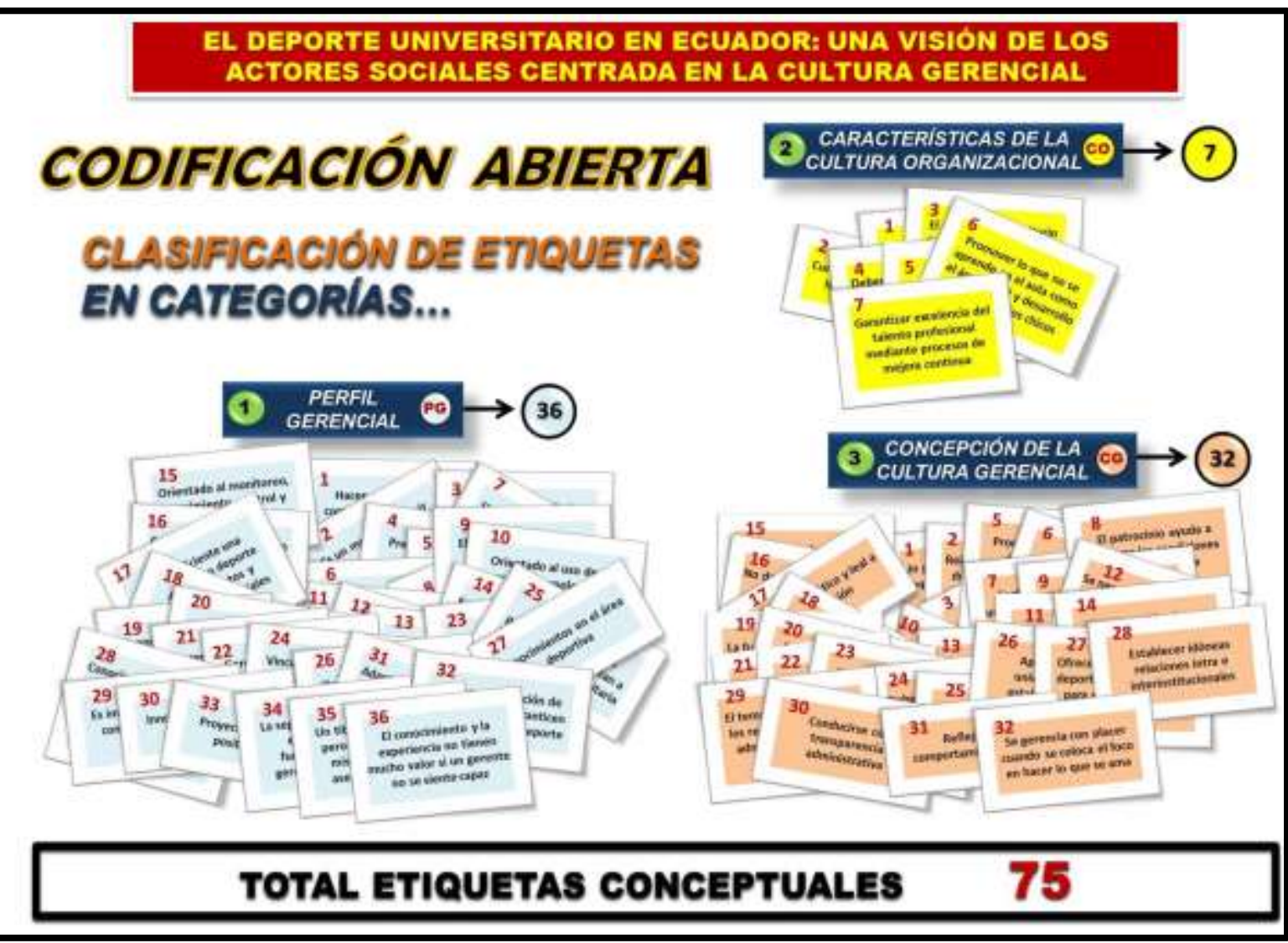

Fuente: Elaboración propia.

Estos datos dan cuenta de la valoración que se otorga a la categoría Perfil Gerencial, mediante la cual se delimita la práctica de una gerencia del deporte universitario que debe fundamentarse en el conocimiento y en la experiencia en el deporte. Respecto al conocimiento, se refiere aquel que está vinculado con el área deportiva y logrado en una institución de educación superior; adicionalmente se desea que el aspirante a ejercer el cargo debiera poseer postgrado y además certificaciones que demuestren capacitación en el deporte y la gerencia. En cuanto a la experiencia en el deporte, se desea de este actor que tenga la necesaria vivencia en el ámbito deportivo, bien sea desde la perspectiva como atleta, entrenador o dirigente, aspecto que ayudaría a comprender e interpretar con precisión las complejidades que engloban al deporte. 
Asimismo, se admite una Concepción de la Cultura Gerencial que pueda combinar un sistema de valores -con foco en aquellos que se ajusten a un comportamiento ético- con ciertas habilidades blandas tanto inter como intrapersonales, a objeto de favorecer relaciones productivas con colaboradores, pares y relacionados. Una concepción de la cultura gerencial que a su vez sirva como agente catalizador de cambios en la gente en sintonía con la misión, visión y valores organizacionales, que en el fondo son factores inherentes a la categoría Características de la Cultura Organizacional.

\section{Tendencias en codificación axial}

Las 75 etiquetas conceptuales identificadas en codificación abierta se cruzaron con sus respectivas categorías en codificación axial, considerando los rasgos distintivos que definen los tipos de relación de la siguiente manera:

- Es una propiedad: Rasgos que identifican y se diferencian del resto de los tipos de relación, caracterizando la esencia de un concepto dentro de una categoría.

- Es una consecuencia: Aquel concepto que se produce como resultado de una acción o hecho derivado de una categoría.

- Es parte: Aun cuando no es un rasgo que lo distingue, es un componente o segmento de un concepto con respecto a la categoría.

De acuerdo a ello, la figura 3 muestra la paridad en la distribución de los datos, donde los tipos de relación "es una propiedad" y "es una consecuencia" tuvieron la misma cantidad de etiquetas enlazadas con 27, dentro de las cuales la categoría Concepción de la Cultura Gerencial obtuvo mayor cantidad de cruces en cada tipo de relación, seguida de Perfil Gerencial. En el caso del tipo de relación "es parte" ( 21 cruces) la categoría Perfil Gerencial destacó ampliamente con respecto a las otras categorías.

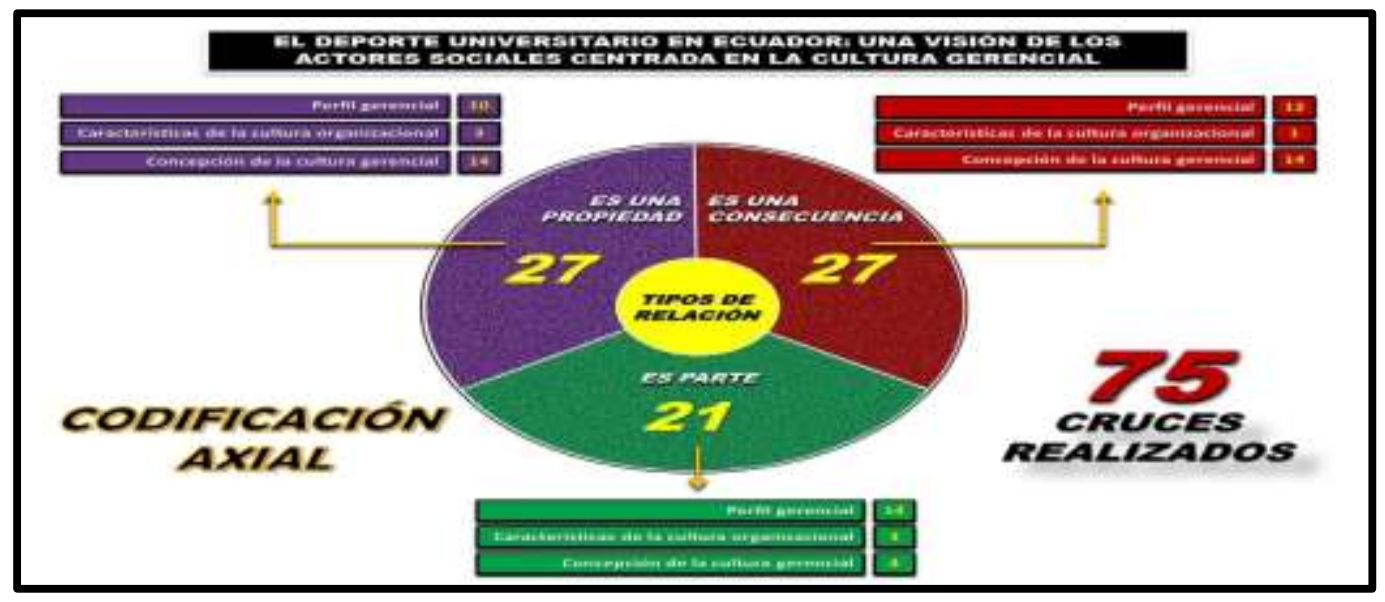

Figura 3. Tendencias en codificación axial.

Fuente: Elaboración propia. 
Estos datos expresan la visión que tienen los actores sociales en torno a una concepción de la cultura gerencial que por naturaleza debiera tener cualidades distintivas que definan la actuación gerencial del deporte universitario, procurando la búsqueda permanente de alternativas para mejorar la gestión como resultado de la interpretación de las necesidades deportivas de la comunidad universitaria y de la sociedad en general. Se presupone como posibles consecuencias de esta concepción de la cultura gerencial, la posibilidad de establecer relaciones con distintos sectores tanto públicos como privados de la sociedad ecuatoriana, con el propósito de asegurar alianzas estratégicas y convenios cooperativos que favorezcan el correcto funcionamiento de las actividades deportivas.

En este mismo orden y dirección, el perfil gerencial debiera considerar como rasgo indivisible a un profesional con formación académica, capacitado y con experiencia en el deporte, cuyo efecto pudiera garantizar en gran medida resultados exitosos a mediano y largo plazo. Finalmente, se destaca el hecho que el manejo de las nuevas tecnologías y las redes sociales para vincularse con la sociedad debiera ser parte del perfil gerencial en un potencial gerente del deporte universitario, y aun cuando no es un rasgo distintivo que lo califica como una propiedad o que lo define como una consecuencia, emerge como un apreciable valor agregado que debe ser considerado en el rol gerencial.

Tal como se ha visto, existe la tendencia por privilegiar a una concepción de la cultura gerencial focalizada en aquellos aspectos que la distinguen y que son de importancia cardinal en la acción gerencial y las probables consecuencias de alto valor que pudiesen derivar de esta acción. En sí, es la aspiración por instaurar un sistema gerencial permeable a todo el deporte universitario, a objeto de configurar un modelo ideal de gerencia soportado igualmente por un sistema valores comunes para todos y que den vida a la cultura gerencial.

\section{Consideraciones acerca de los resultados}

Sobre la base de los planteamientos anteriores, donde se determinó las tendencias en codificación abierta y codificación axial producto del riguroso y detallado análisis ajustado a la aplicación del método comparativo constante para garantizar la recurrente revisión y comparación de los datos que emergieron en el proceso investigativo, se continuó con el enfoque interpretativo de los hallazgos a los fines de dar respuesta a la visión y alcance de la cultura gerencial del deporte universitario en Ecuador. En este tenor, a continuación se exponen los resultados obtenidos en cada una de las categorías seleccionadas en este estudio: (a) Perfil gerencial; (b) Características de la cultura organizacional; y (c) Concepción de la cultura gerencial.

\section{Perfil Gerencial}

Se deduce de los datos que se derivan de este análisis, la prevalencia que se confiere al candidato ideal para conducir el deporte universitario con un perfil gerencial sustentado en 
el conocimiento, esto es aquel que se logra en una universidad donde se otorgue el grado de licenciado en el área deportiva o carreras afines, por ejemplo en aquellas vinculadas a las ciencias aplicadas al deporte. Asimismo, el aspirante a ejercer el quehacer gerencial en este sector debe respaldar este conocimiento con estudios de cuarto nivel, preferiblemente en postgrados adscritos a las ciencias administrativas y gerenciales, conjuntamente con certificaciones que demuestren capacitación sistemática y continua en el área deportiva y gerencial.

Este conocimiento, -que además de contribuir con el desarrollo de saberes para crear valor utilitario en el área deportiva, otorga una certificación con reconocimiento y da prestigio social- debe combinarse con la capacidad de liderazgo para relacionarse con otros, para comunicarse con asertividad, para motivar a la gente, para compartir información de valor, para delegar tareas; en sí, es la capacidad que debe tener un gerente para ejercer un liderazgo que dinamice una labor coordinada con el conjunto de personas bajo su conducción a fin de asegurar el cumplimiento de los objetivos y metas que deban ser programadas.

Cabe agregar, que aun cuando se valora el conocimiento logrado a través de la formación académica y los procesos de capacitación requeridos para el ejercicio del rol, se estima como condición un actor-gerente con la vivencia en el deporte -bien sea como atleta, entrenador o dirigente del deporte-. Esta vivencia permitiría conocer de primera mano las necesidades que demanda el deporte universitario, a los fines de aportar soluciones rápidas y oportunas, cónsonas con los resultados de excelencia y calidad que desea este sector deportivo.

En las mismas circunstancias, un gerente del deporte universitario debe ser capaz de manejar con propiedad los procesos administrativos y estar orientado al seguimiento, monitoreo, control y evaluación de la gestión, a fin de anticipar posibles desperdicios, ofrecer retroalimentación oportuna y reorientar acciones que no estén alineadas con los objetivos y metas que sean planteados. Se aspira entonces un actor-gerente del deporte tendente a considerar qué mecanismos de control de gestión han sido exitosos, cuáles han fallado, qué debe mantenerse, qué se debe actualizar; en síntesis, mecanismos permanentes de control y evaluación de la gestión para evolucionar.

Visto así, un gerente del deporte universitario debe idear, elaborar, diseñar planes prospectivos y tener la capacidad para desarrollar y ejecutar estrategias deportivas con impacto, soportadas en indicadores mensurables que le permitan por un lado detectar los problemas macros, mesos y micros en el campo deportivo, y por otro lado conocer el estatus de avance de las acciones realizadas. En este contexto, es menester poseer visión a largo plazo, planificando más allá de una gestión, sin descuidar el corto y mediano plazo, teniendo el talante para apoyarse en referencias exitosas de otros países.

Todo lo anterior debe conjugarse con un sistema de valores alineados con la filosofía de la universidad, plataforma significativa que ayudaría -mediante el modelaje y la influencia del 
gerente- a guiar el comportamiento de las personas adscritas a la unidad deportiva y al mismo tiempo a proyectar una imagen positiva del rol. Un comportamiento centrado en valores que también promueva su vinculación con los problemas que afecten a la población universitaria con el porpósito de ofrecer posibles soluciones desde la perspectiva deportiva.

\section{Características de la Cultura Organizacional}

En relación con las características de la cultura organizacional, si bien hubo en esta categoría una brecha apreciable en la producción de etiquetas conceptuales respecto a la precitada y a la subsiguiente categoría dado al interés mostrado hacia el constructo gerencia, se destaca acá la consideración que se presta a la filosofía organizacional -misión y visión- como plataforma integradora de la cultura organizacional del deporte universitario, matizada por las creencias, los valores y las normas, las cuales van a condicionar las actitudes y los comportamientos de las personas, factores que resultan congruentes con los postulados de Robbins (2004) quien señala que "...la cultura organizacional sirve como un mecanismo que crea sentido y permite el control, que orienta y da forma a las actitudes y comportamientos de los empleados" (p. 528).

De acuerdo con ello, un gerente del deporte universitario sería en su esencia un factor crítico para inducir los cambios en las actitudes y en el comportamiento requerido en el ejercicio del rol gerencial y en los miembros del equipo, con la finalidad de dar direccionalidad a la cultura organizacional mediante acciones que conduzcan a un desempeño exitoso, congruente con la filosofía organizacional en resguardo de la eficiencia administrativa y la eficacia en los resultados, donde el elemento catalizador de estos cambios sería el modelaje de valores. Ello implicaría integrar de manera permanente los valores en las funciones, responsabilidades y tareas, introducir los valores en cada proceso, comunicar los valores de manera formal e informal; en fin, es la práctica de valores de manera permanente en vez de proclamarlos, actuando con rectitud, consideración y respeto, con la intención de asegurar una sólida cultura organizacional.

En relación con esto último, cabe agregar la significación que de igual modo se otorga a los valores como factor integrador de la comunidad universitaria a través del deporte, principalmente a aquellos que promueven la unidad, la solidaridad y la equidad, en atención a las particularidades de Ecuador, país que se caracteriza por ser pluricultural, multiétnico, con distintos estratos sociales y con diversidad de géneros, tal como lo afirma un entrevistado: "Se debe ser más incluyente y trabajar en el bienestar de los estudiantes universitarios, promover lo que no se aprende en el aula como el área social y desarrollo integral de los chicos a través de las actividades deportivas".

Adicionalmente, se acentúa la atención que debe prestarse a los procesos de adiestramiento y capacitación de la labor gerencial y aguas abajo en todo el personal, a fin de propiciar la optimización de la gestión deportiva mediante la adopción de conocimientos y herramientas 
que propicien un desempeño superior, en la búsqueda de una mayor y mejor efectividad organizacional para el logro de los objetivos propuestos.

Son factores que sin duda corresponden a una cultura organizacional fruto de la percepción que de ella tienen los actores sociales. Una cultura organizacional que pueda soportarse en firmes valores susceptibles de ser compartidos por la comunidad deportiva y por extensión por toda la comunidad universitaria. En definitiva, una cultura organizacional que pueda capitalizarse en la necesaria cohesión entre los distintos grupos de interés, en la anhelada identificación institucional y en la esperada satisfacción laboral.

\section{Concepción de la Cultura Gerencial}

En lo atinente a la concepción de la cultura gerencial, categoría que se ponderó con una importante cantidad de etiquetas conceptuales, los datos revelan la inclinación por delinear un comportamiento gerencial en el deporte universitario que pueda ser entendido y compartido por los miembros del equipo, dando significado a las acciones, objetivos y metas a seguir. En este sentido, se demanda una cultura gerencial que esté soportada en sólidos valores éticos y morales que ayuden a conducirse de manera justa, honesta, respetuosa, equitativa y correcta con el personal en cualquier circunstancia, en el interés de crear un ambiente de confianza, asegurar comportamientos productivos y propiciar un buen clima laboral.

Así pues, son valores que además de guiar el comportamiento de las personas, se deben conjugar con ciertas habilidades blandas, que si bien se describen en la categoría perfil gerencial aludiendo a la capacidad de liderazgo, son de índole interpersonal, siendo también pertinente que un gerente del deporte universitario posea habilidades intrapersonales para responder con efectividad, tales como:

- La necesaria consciencia para reconocer sus fortalezas y debilidades, estando abierto a la retroalimentación.

- El reconocimiento de las propias emociones y sus efectos sobre el desempeño.

- El autocontrol para manejar las desavenencias y conflictos.

- La flexibilidad para entender y orientar los cambios.

- La confianza para creer en sí mismo, asumir riesgos, nuevos retos y habilidades.

- La fiabilidad para actuar con ética, crear confianza y admitir sus errores, al tiempo que confronta las acciones no éticas en los demás.

En suma, estos valores y habilidades inter e intrapersonales trastocan aspectos actitudinales, axiológicos y emocionales; habilidades que Alles (2011) las define como competencias, 
vistas ellas como “... características de personalidad, devenidas en comportamientos, que generan un desempeño exitoso en un puesto de trabajo" (p. 33). Es evidente entonces el interés manifiesto por una concepción de la cultura gerencial que pueda articular el conocimiento -por demás accesible y apropiadamente gestionado- con las competencias requeridas para garantizar un desempeño superior.

En este orden de ideas, la cultura gerencial del deporte universitario debe fundamentarse en el indispensable liderazgo como se ha enfatizado, el cual debiera inspirar a los miembros del equipo para superar expectativas, fomentar la búsqueda de soluciones, anticipar situaciones de conflicto, tomar decisiones rápidas y oportunas. En sí, un liderazgo que impulse una gestión centrada en resultados. Para que esto pueda ser posible, es imperativo que exista amor hacia el deporte, la necesaria pasión para emprender acciones que impulsen la organización y desarrollo de eventos deportivos con excelencia y de igual manera otorguen beneficios y satisfacción de la comunidad universitaria. Ello también implica la disposición por empoderar a la gente, dando espacios a aquel que posea elevado desempeño y promoviendo procesos de capacitación que ayuden a mejorar la gestión deportiva.

Desde luego, materializar este conjunto de aspectos exigiría el fortalecimiento de relaciones con organismos deportivos y de sectores que resulten estratégicos -públicos y privados en ambos casos-, coordinando encuentros con cada una de las instancias a fin de establecer alianzas y convenios que involucren el patrocinio económico o de dotación de insumos para la organización de eventos deportivos entre institutos, escuelas politécnicas y universidades, así como la cobertura televisiva y de otros medios de comunicación que ayuden a la difusión del deporte universitario. En compensación, las instalaciones deportivas -que debieran comercializarse- estarían contempladas en los acuerdos, por ejemplo la posibilidad de colocar vallas publicitarias en los espacios deportivos y el alquiler de sus instalaciones a bajos costos o su exoneración, dependiendo de los acuerdos alcanzados. Serían alianzas y convenios con el sector público y privado que sin lugar a dudas generarían recursos adicionales y contribuirían al fortalecimiento del sistema deportivo universitario.

Significa entonces la concepción de una cultura gerencial congruente con la majestad del cargo, siendo proactivo ante iniciativas comerciales y de patrocinio deportivo, postura que resalta un entrevistado de esta manera: "se debe saber gestionar a fin de generar recursos, no solo esperar recursos". Después de todo, es ofrecer opciones creativas, buscar ideas, y también equivocarse para aprender y seguir avanzando en aras de obtener recursos sin la necesidad de esperar a que estos lleguen, porque a fin de cuentas gerenciar el deporte universitario debe ser una vocación, sobre todo en un contexto de país donde los recursos para el deporte en general son escasos.

\section{Conclusiones}


Las evidencias que surgieron en esta investigación, permitieron descubrir hallazgos significativos en torno a la visión y alcance de la cultura gerencial del deporte universitario en Ecuador. Son hallazgos que dan cuenta de las creencias que se tienen de la cultura gerencial como elemento intrínseco en la actuación de quien se estima debe conducir el deporte en cualquier institución de educación superior. En efecto, una cultura gerencial centrada en las necesidades e intereses de la comunidad universitaria y en la construcción de una organización deportiva unificada, abordando los problemas y las interrelaciones personales con la efectividad esperada.

Bajo esta premisa, se desprende de las categorías de análisis seleccionadas en este estudio, la importancia que se otorga a un liderazgo gerencial que logre la consciencia y mística de grupo en una gestión deportiva, debiendo sustentarse en un sistema de valores que sirva de plataforma para guiar la actuación de las personas; un liderazgo gerencial y un sistema de valores que en definitiva den direccionalidad al conjunto de acciones, objetivos y metas en correcta alineación con la filosofía universitaria.

Así pues, liderazgo y valores destacan indefectiblemente como conceptos representativos de la cultura gerencial, una suerte de ejes donde se enlazan elementos intangibles que emergieron en el proceso investigativo vinculados con las habilidades intra e interpersonales; liderazgo y valores que en sí mismos se configuran como competencias deseables en la dinámica gerencial y que deben armonizarse con el conocimiento logrado en la academia, en los procesos de capacitación y en la experiencia en el deporte. En resumidas cuentas, una cultura gerencial con gran influencia en el comportamiento de la gente que impulse la cohesión, la lealtad y el compromiso con el deporte universitario.

Como corolario a lo anterior, se desea dejar constancia que es necesario abordar una investigación orientada a analizar cuál es la cultura gerencial en escenarios reales y cómo son los patrones de comportamiento gerencial que comúnmente operan en el deporte universitario, a los fines de poder contrastarlos con los hallazgos encontrados en este estudio, estimando las brechas de aquello que se desea con lo que acontece en la realidad empírica.

\section{Referencias Bibliográficas}

Aguilar, P. (2014). Gerencia del deporte de alto rendimiento en Venezuela: hacia una implantación efectiva de los procesos administrativos. Saarbrücken: Editorial Publicia.

Alles, M. (2001). Desarrollo del talento humano basado en competencias. Buenos Aires: Granica.

Arias, F. (2016). El Problema de Investigación: Introducción a la Metodología Científica. Caracas: Editorial Episteme. 
Corbin, J. y Strauss, A. (2002). Bases de la investigación cualitativa: Técnicas y procedimientos para desarrollar la teoría fundamentada. Medellín: Universidad de Antioquia.

Domínguez, J. (2018). Pastillas gerenciales. Chimbote: Universidad Católica Los Ángeles de Chimbote.

Echeverría, R. (2009). Ontología del lenguaje. Buenos Aires: Ediciones Granica.

Gómez, S. (2018). Rasgos distintivos de la cultura gerencial en las organizaciones del deporte: El caso Ecuador. Revista Digital de Educación Física EmásF, 9 (52), 3549.

Gómez, S. (2017a). Gerencia estratégica en organizaciones del deporte. En: Yanez, P. y Gómez, S. (Eds.). (2017). Gerencia del deporte en el siglo XXI: Modelos, estrategias, alcances y retos, 36-51. Quito: Offset Chávez.

Gómez, S. (2017b). Cultura gerencial en las organizaciones del deporte: Valores, creencias, prácticas, herramientas, métodos y procedimientos en organizaciones del deporte. Saarbrücken: Editorial Académica Española.

Martínez, M. (2009). Ciencia y arte en la metodología cualitativa. México: Trillas.

Morin, E. (1989). La noción de sujeto, nuevos paradigmas, cultura y subjetividad. Barcelona: Paidos.

Rivera, D.; Carrillo, S.; Forgiony, J.; Nuván, I. y Rozo, A. (2018). Cultura organizacional, retos y desafíos para las organizaciones saludables. Revista Espacios, 9 (22), 27 40.

Robbins, S. (2004). Comportamiento organizacional. México: Pearson Educación.

Rusque, A. (2007). De la diversidad a la unidad en la investigación cualitativa. Caracas: Vadell.

Rusque, A. (2008). Empresarios y gerentes: sujetos en formación en la educación superior. Cuadernos de Postgrado: Gerencia y Condición Humana, 28, 9-23.

Santillán, M. (2010). Gestión del conocimiento. El modelo de gestión de empresa del siglo XXI. Barcelona: Gesbiblo.

Swiringa J. y Wierdma, A. (1995). La Organización que aprende. Addison-Wesley Iberoamericana: Estados Unidos. 
Taylor, M. (2007). El desafío de las diferencias culturales para las relaciones públicas globales. En Pendertgrast, M. (Coord.), Grandes Casos Empresariales, (pp. 133157). Barcelona: Deusto.

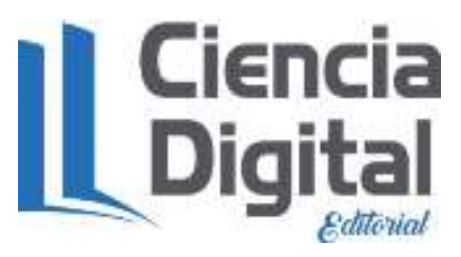

\section{PARA CITAR EL ARTÍCULO INDEXADO.}

Gómez Barrios, S. (2019). Cultura gerencial: visión y alcances en el deporte universitario. Ciencia Digital, 3(2.5), 59-76. https://doi.org/10.33262/cienciadigital.v3i2.5.531

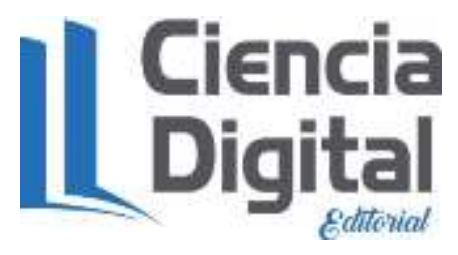

El artículo que se publica es de exclusiva responsabilidad de los autores y no necesariamente reflejan el pensamiento de la Revista Ciencia Digital.

El artículo queda en propiedad de la revista y, por tanto, su publicación parcial y/o total en otro medio tiene que ser autorizado por el director de la Revista Ciencia Digital.
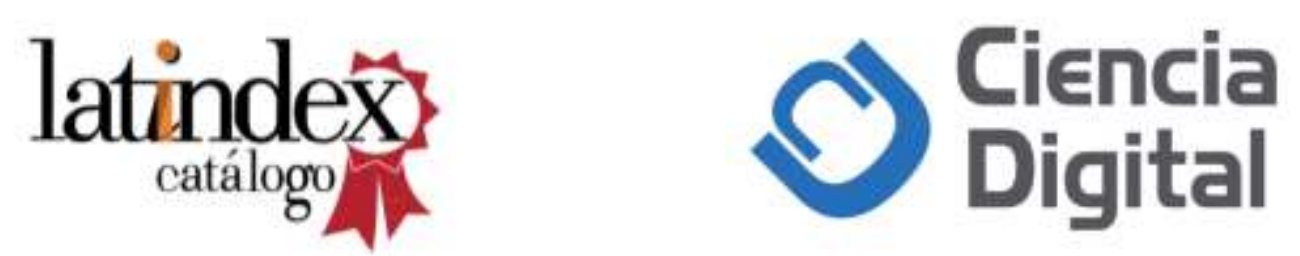